\title{
Écosystème
}

\section{LU, VU ET ENTENDU}

\section{Léo Guy-Denarcy}

Volume 1, numéro 1, 2018

Musique

URI : https://id.erudit.org/iderudit/1058619ar

DOI : https://doi.org/10.7202/1058619ar

Aller au sommaire du numéro

Éditeur(s)

La chambre blanche

ISSN

2562-3222 (numérique)

Découvrir la revue

Citer cet article

Guy-Denarcy, L. (2018). LU, VU ET ENTENDU. Écosystème, 1(1), 27-32.

https://doi.org/10.7202/1058619ar

Ce document est protégé par la loi sur le droit d'auteur. L'utilisation des services d'Érudit (y compris la reproduction) est assujettie à sa politique d'utilisation que vous pouvez consulter en ligne.

https://apropos.erudit.org/fr/usagers/politique-dutilisation/
Cet article est diffusé et préservé par Érudit.

Érudit est un consortium interuniversitaire sans but lucratif composé de l'Université de Montréal, l'Université Laval et l'Université du Québec à Montréal. Il a pour mission la promotion et la valorisation de la recherche. https://www.erudit.org/fr/ 


\section{LU, VUET ENTENDU}

\section{Léo Guy-Denarcy}

Depuis l'exposition Sons et Lumières au Centre Georges Pompidou ${ }^{43}$, il paraît nécessaire de penser à nouveau les dimensions muséales de l'implication phonographique dans le champ des arts plastiques. Pour travailler à l'étude de cette mise en relation, il semble opportun de faire dialoguer le travail de deux artistes à la croisée de ces deux disciplines. Il s'agit premièrement du travail de Christian Marclay né en 1955 à San Rafael ${ }^{44}$ et du travail expérimental et bruitiste de Jean-Louis $\operatorname{Costes}^{45}$ également né en 1955 à Paris. Pris séparément, les deux travaux en présence ne suggèrent pas de connexion logique hors des liens qu'ils construisent entre arts plastiques et démarches sonores. À l'expérimentation parfois proto-photographique de Christian Marclay ne répond pas la "mise en œuvre » trash d'une vie de performeur singulier à l'image de Jean-Louis Costes. Si leurs travaux respectifs croisent des problématiques liées au son et à la performance, il est nécessaire de noter que leurs réseaux de diffusion, leurs carrières et leurs domaines d'expérimentation sont aujourd'hui radicalement différents. Il demeure cependant une réponse chronologiquement datée à une problématique en pleine effervescence dans les années 2002-2010. En effet, si l'exposition Sons et lumières fait date dans l'étude historique et technique du lien entre les pratiques sonores et les arts visuels, cette dernière marque également l'ouverture d'une période empreinte de nombreuses recherches et connections interdisciplinaires. Nous pouvons citer entre autres la naissance de la revue Volume! (2002), la redécouverte du travail plastique d'Alan Vega (2009) et de Laurie Anderson (2010), la forte exposition des travaux de Dominique Blais et Pierre-Laurent Cassière ou encore l'exposition Playback au Musée d'Art Moderne de la Ville de Paris en 2007. La liste pourrait s'allonger considérablement.

Si nous ne pouvons donner avec assurance les raisons d'un retour à cette période de la question du son et de ses représentations visuelles, il est possible de souligner quelques interrogations, publications et traductions majeures qui ont accompagné ce débat durant quelques années. Premièrement, et de manière évidente, l'exposition Sons et Lumières du Centre Georges Pompidou à Paris en 2005 marque une étape dans cette réflexion notamment dans sa filiation directe avec l'exposition Dyonisiac ${ }^{46}$. Nous pouvons, à la suite

43 Sophie Duplaix et Marcella Lista, Sons \& Lumières : Une histoire du son dans l'art du XXe siècle, Éditions du Centre Georges Pompidou, Paris, 2005.

44 Nous nous intéressons particulièrement ici aux performances interprétées par Christian Marclay dans les années 80 à Boston et New York, puis aux interprétations des oeuvres Graffiti Composition ou Shuffle réalisées notamment en 2008 et 2009 à Rennes et Paris.

45 Jean-Louis Costes est musicien performeur et écrivain. Il explore depuis 1983 une variété de formats sonores et visuels souvent qualifiés de transgressifs.

46 Dionysiac, commissaire Christine Macel, Centre Georges Pompidou, Paris, 2005. L'exposition est présentée comme une exposition-réflexion qui mêle artiste reconnus et émergents. Le travail de mise en relation autour des pratiques de Jason Rhoades, Paul McCarthy ou encore Jonathan Meese a insufflé un parfum de scandale dans l'exposition. La 
de ce travail, évoquer la traduction en français de l'ouvrage L'Espace de la galerie et son idéologie $^{47}$ de Brian O'Doherty qui stimulent nombre d'étudiants, d'artistes, de commissaires d'exposition et de critiques d'art jusqu'à aujourd'hui. Enfin, le retour sur le devant de la scène du mouvement punk angelinos à travers notamment l'oeuvre du groupe Destroy all monsters et construit autour des artistes Mike Kelley et Jim Shaw traduit également de ce phénomène. Ces trois faisceaux divergents témoignent d'un souci attendu pour ce qui se fait principalement extérieur à la scène artistique dans son acception convenue et dans une cartographie de plus en plus précise de la création contemporaine au lendemain des années 90 . Il est alors de mise de rechercher et de trouver ce qui se fait extérieur au territoire nommé de l'art contemporain lui-même évoluant dans une acception qui semble à ce moment de plus en plus réduite.

Alors, si la question du son et de sa représentation visuelle occupe une place centrale et constitutive dans l'oeuvre de Christian Marclay et Jean-Louis Costes, c'est d'abord par l'utilisation du mixage, par l'expérimentation visuelle de la partition puis par le lien qui se constitue entre composition et langage dans un espace possible, mais aussi collectif. Il semble alors que ces travaux se situent (paradoxalement) dans la recherche d'un espace extérieur et prolifique à un champ constitué et au statut d'auteur.

Ainsi, l'enjeu historique d'une musique plastique et de son appréciation nous amène à questionner chez les deux auteurs en présence un bornage de l'exposition et des pratiques plastiques et artistiques. Cette injonction à l'écoute et à l'appréciation de la matérialité sonore s'est également formée autour de pratiques collectives qui semblent aujourd'hui répondre à des problématiques ultra-contemporaines.

Une étude des oeuvres plastiques et sonores de Costes et Marclay, et de leur émergence, nous permet de constater que tous deux sont marqués par la scène punk-noise des années 70/80 et par la recherche de ce " hors cadre », hors scène (littéralement l'ob-scène) de la musique dans son expression et son appréciation. Cette dimension radicale doit se concrétiser dans une rupture des espaces d'interprétation de la musique. Celle-ci se trouve matérialisée par la galerie d'art, le musée, la cave ou les bars, mais aussi dans une séparation du corps du musicien et de la production du son. Ne plus « faire » de la musique sur scène ni l'interpréter devient alors une revendication qui permet d'occuper l'espace autrement en se marquant ainsi dans une évidente filiation du 4'33" de John Cage et son très relatif silence. Le silence donc, mais aussi le jeu des performeurs comme l'image du sonore et de son expression deviennent le lieu de cette libération des protagonistes et de l'espace. Ces derniers vont prendre différentes formes: de mises en scène violentes et parfois scatologiques chez Costes elles se font chez Marclay une injonction au visuel, au silence ou à l'image d'un corps qui ingurgite la musique (Fast Musique 1981), mais aussi à la

musique par la présence d'une sound room est omniprésente soulignant déjà les liens qui se tissent entre les pratiques des artistes de cette génération.

47 Brian O’Doherty, White Cube : L'espace de la galerie et son idéologie, Éditions JRP Ringier, coéditions Maison Rouge, Paris, 2008, Trad. Patricia Falguieres. 
destruction du support dans le cadre de ces interprétations ${ }^{48}$. Ces démarches sont en tout point le lieu de l'intellectualisation d'un geste musical spontané qui vise à sublimer le geste de l'auteur (du producteur de son) afin de provoquer chez le spectateur une interrogation et parfois une étrange pulsion scopique qui se matérialise dans la mise en valeur d'un bruit, de l'aléatoire extérieur au proprement musical : il s'agit alors de la mastication, du choc ou du coup comme du $\mathrm{cri}^{49}$.

La spatialisation de la pratique musicale et bruitiste trouve un écho particulier dans la scénographie et sa contemporanéité et elle nécessite la mise en perspective de quelques démarches antérieures. Il s'agit pour Costes d'une introduction à la manière du groupe Fluxus de l'élément musical dans ce qu'il qualifie d'opéra (dans son appréciation totale) eu égard à ses performances et qui situe son oeuvre dans l'héritage du théâtre filmé, parfois pour l'objectif de la caméra uniquement. Dans le cas de Marclay, la trajectoire depuis les " concerts » bruitistes vers une mise en exergue d'un son in abstentia fait écho à son incapacité à jouer d'un instrument et à une volonté de développer l'improvisation puis de travailler depuis l'élément sonore.

En recherchant pour l'écoute et pour le son un espace propre et une scénographie, les deux artistes répondent à l'interrogation des modes de production de l'exposition à la fois topographiques, « légaux », mais aussi symboliques et marqués par le " comment faire ». Ils s'inscrivent également dans un questionnement des modes de production sociaux de l'art et de ses territoires. Deux oeuvres cristallisent historiquement ces questions topographiques. La première est le Museum highlights et la très particulière «visite guidée» du Musée de Philadelphie proposée par Andrea Fraser en $1989^{50}$. L'oeuvre annonce par sa lecture sociale et psychosociologique, ce qui va fonder les interrogations à venir sur le musée et son contenu. En effet, cette visite se trouvant au sein d'un espace symbolique et marqué se trouve être l'exacte contemporaine de grandes enquêtes lancées par les historiens et les sociologues sur l'institution muséale et qui annoncent de même la critique institutionnelle. La seconde pièce est l'oeuvre présentée par Marcel Broodthaers à la Documenta $\mathrm{V}$ de Kassel et qui vient borner un espace privé (visible) et muet au sein des espaces d'exposition. L'oeuvre se situe dans la section Art Moderne du département des Aigles et il est particulièrement saisissant que ce jeu à la fois poétique (par sa taille réduite) soit également une réponse à la circulation dans l'espace du musée et semble s'ouvrir à ce que Wilfried Dickhoff nomme « une conquête de l'espace. ${ }^{51}$ » Cette conquête marque avant tout une volonté de soumettre le cadre muséal comme le feront quelques années plus tard

\footnotetext{
48 Lors de plusieurs performances, Christian Marclay fait jouer des vinyles qu'il a préalablement brisés (Circa, 1983) ou encore qu'il a disposés au sol pour que le public endommage le support avant sa diffusion (Kitchen 1982).

49 Marianne Massin, «Quiet please, work in progress. Faire silence : de l'injonction à son retournement », dans Christian Marclay Snap!, Les Presses du réel, Dijon, 2009.

50 Andrea Fraser, Museum Highlights : A Gallery Talk, 1989, U-Matic PAL, couleur, son, consulté au Centre Georges Pompidou.

51 Wilfried Dickhoff, «La Conquête de l'espace » dans Marcel Broodthaers Livre d'images, Flammarion, Paris, 2013.
} 
Costes et Marclay dans leurs travaux scéniques et sonores, mais aussi d'y introduire un propos en absence comme un contenu tierce.

La spatialisation de ces travaux comme l'expérience des oeuvres nous conduisent à interroger ces expériences esthétiques sous un angle nouveau par la lecture du lieu de l'exposition et du bornage auxquelles celles-ci nous conduisent. En effet, au-delà d'une notion juridique, géographique et spatiale cette notion nous invite à imaginer l'existence d'une sphère autonome dans le champ artistique ou plutôt de sa constitution au sein même de ce que Alois Riegl nomme la Kunstindustrie, c'est-à-dire la distinction entre le monument (le lieu) et le monument historique (sa portée symbolique). Cette double appréciation des lieux est particulièrement sensible dans le cas des travaux sonores. Les travaux de Christian Marclay ont été souvent rapprochés, ou du moins mis en filiation, des oeuvres de Marcel Duchamps et de John Cage, deux figures singulières de l'histoire de l'art qui marquent une forte implication de l'interdisciplinarité et d'une ambition collective dans le champ de la création. Marclay s'inscrit, à la faveur d'une bourse d'échange entre le MCA de Boston et la Cooper Union, dans une scène artistique portée par deux phénomènes. Tout d'abord une connivence entre les mondes de la scène et les arts visuels puis par la nouvelle émergence des pratiques photographiques et leur exposition. De la même manière, les débuts de Costes sont marqués par la rencontre de la scène noise avec la production artistique et scénique dite «indue», elle-même empreinte des nouvelles possibilités de montages visuels et sonores ${ }^{52}$. Le mouvement est structuré autour de thèmes provocateurs et par les organisations antifascistes, les revendications politiques et sociales, mais aussi une expression visuelle violente et radicale. Le travail de dessin de Costes est durablement marqué par ce mouvement auquel s'ajoute un humour et un second degré très personnel. Principalement diffusé dans les marges de la scène underground, le travail de Costes et Marclay s'extrait d'une dimension minoritaire par une nouvelle appréciation critique au début des années 2000, mais, encore une fois, les deux oeuvres se diffusent et se font connaître dans des milieux radicalement différents. Les recherches artistiques de cette période prolongent une remise à plat du statut de l'artiste et de l'auteur par la rupture des codes et des conventions sonores et visuelles et du lieu de leur appréciation. Comme l'énonce Patricia Falguières, l'ouverture vers les marges « où les artistes eux-mêmes n'ont cessé d'introduire dans ce jeu (de l'histoire de l'art) des objets et des gestes qui n'en relevaient pas » a construit les avant-gardes, mais aussi l'élargissement des bornes de l'analyse artistique sociohistorique ${ }^{53}$.

Néanmoins, il semblerait que dans cette démarche de recherche artistique propre à la plasticité et à la «sonorité », les artistes en présence ne demeurent pas dans un schéma individuel de création. En effet, l'histoire et donc l'histoire de l'art, est marquée notamment

52 Voir à ce propos Lisa Carver et al., L'Art brutal de Jean-Louis Costes, Éditions Exposition radicale, Paris, 2012.

53 La parole au Centre, selon Patricia Falguière «L'histoire de l'art par ces artistes, même », Centre Georges Pompidou, Paris, 2011. 
dans sa dimension musicale et sonore, par l'implication du collectif dans la conception et la réalisation des oeuvres. Il suffit alors d'évoquer l'implication des groupes Fluxus, Ecart, des mouvements punk-noise ou encore de Art \& Language pour percevoir la dimension collective qui leur incombe. Il semblerait également qu'une réponse aux problématiques artistiques liant les formes visuelles et bruitistes réside dans leur mode de production et dans l'implication collective du travail artistique ${ }^{54}$. La généalogie du travail sonore nous conduit également à regarder ce que dit le collectif dans la manière de travailler. Il ne s'agit pas ici d'entreprendre une étude exhaustive des démarches collectives sonores et visuelles. Cette étude ne serait pas vraisemblable et comme le remarquait Irit Rogoff : une étude ou une exposition volontairement exhaustive sur les collectifs d'artistes ne serait pas possible.

Il est toujours frappant de constater que le monde occidental se prend à rêver de travaux collectifs, collaboratifs, mais aussi pluridisciplinaires à chaque âge marqué par l'idéalisme et le renouveau politique. Cette démarche se retrouve dans la vague bruitiste initiée par Luigi Russolo qui avec son ouvrage L'Art des bruits ${ }^{55}$ marque durablement le futurisme italien. Une montée de fièvre collective et collectiviste imprègne également les avantgardes russes des années 20 et notamment le constructivisme empreint d'une modernité sonore liée aux avancements technologiques du début du $\mathrm{XX}^{\mathrm{e}}$ siècle. Les points de jonction entre les collectifs d'artistes visuels et sonores persistent au sein de l'ensemble des stratégies qui ont été et continuent à être influencées par la critique des hiérarchies culturelles, telles qu'elles ont notamment été articulées dans les années 60 par l'élaboration de nouveaux discours sur les genres et sur les races. Les stratégies en question sont notamment une lutte pour changer, inverser et trouver une issue pour entrer ou sortir du cadre muséal et institutionnel. Les travaux de Christian Marclay et Jean-Louis Costes ont toujours souhaité remettre en question le lieu de production de la musique comme des arts visuels, mais aussi leur mode d'interprétation. Nous avons pu le voir, les deux artistes se construisent bien plus sur une recherche de l'espace de diffusion, d'appréciation et d'expression que sur une connaissance et une maîtrise instrumentale. Il y aurait alors dans la mise en scène de ces travaux le résultat d'une véritable expression libératrice du son dans les arts visuels démultipliant les instruments (phonographes, acteurs, musiciens), mais aussi les collaborations pour construire des expériences uniques dans leurs multiplicités. Ce paradoxe, qui appartient en réalité à une histoire du temps long et que nous retrouvons aujourd'hui comme une montée en puissance des activités collectives de travail (tant du point de vue sonore que visuel), a débuté il y a 50 ans à une période où l'activité artistique avait le désir d'insuffler un idéalisme social combiné à une volonté d'articuler le travail artistique dans la société.

\footnotetext{
54 Il est intéressant de ce point de vue de noter la démarche curatoriale de Christian Marclay pour la Biennale de Venise 2017 au sein du pavillon français en collaboration avec Lionel Bovier pour le projet Echoes of the Studio de Xavier Veilhan : «un dispositif musical dans lequel des musiciens professionnels du monde entier, invités par l'artiste et les commissaires du pavillon interviendront pendant toute la durée de la manifestation », Institut Français.

55 Luigi Russollo, L'Art des bruits, Éditions Allia, Paris, 2003.
} 


\section{Références}

CARVER, Lisa et al. L'Art brutal de Jean-Louis Costes, Éditions Exposition radicale, Paris, 2012. 224 p.

DICKHOFF, Wilfried, « La Conquête de l'espace » dans Marcel Broodthaers : Livre d'images, Éditions Flammarion, Paris, 2013. 319 p.

DUPLAIX, Sophie et Marcella Lista. Sons \& Lumières : Une histoire du son dans l'art du XXe siècle, Éditions Centre Georges Pompidou, Paris, 2005. 400 p.

MASSIN, Marianne, « Quiet please, work in progress. Faire silence : de l'injonction à son retournement », dans Christian Marclay Snap!, Éditions Les Presses du réel, Dijon, 2009. $368 \mathrm{p}$.

O'DOHERTY, Brian. White Cube : L'Espace de la galerie et son idéologie, Éditions JRP Ringier, Coéditions Maison Rouge, trad. par Patricia Falguières, 2008. 208 p.

RUSSOLLO, Luigi, L'Art des bruits, Éditions Allia, Paris, 2003. 40 p. 